\title{
Guillain-Barré syndrome: The first documented COVID-19-triggered autoimmune neurologic disease
}

\author{
More to come with myositis in the offing
}

Marinos C. Dalakas, MD

Neurol Neuroimmunol Neuroinflamm 2020;7:e781. doi:10.1212/NXI.0000000000000781

\author{
Correspondence \\ Dr. Dalakas \\ marinos.dalakas@jefferson.edu
}

\section{MORE ONLINE}

Abstract

\section{Objective}

To present the COVID-19-associated GBS, the prototypic viral-triggered autoimmune disease, in the context of other emerging COVID-19-triggered autoimmunities, and discuss potential concerns with ongoing neuroimmunotherapies.

\section{Methods}

Eleven GBS cases in four key COVID-19 hotspots are discussed regarding presenting symptoms, response to therapies and cross-reactivity of COVID spike proteins with nerve glycolipids. Emerging cases of COVID-19-triggered autoimmune necrotizing myositis (NAM) and encephalopathies are also reviewed in the context of viral invasion, autoimmunity and ongoing immunotherapies.

\section{Results}

Collective data indicate that in this pandemic any patient presenting with an acute paralytic disease-like GBS, encephalomyelitis or myositis-even without systemic symptoms, may represent the first manifestation of COVID-19. Anosmia, ageusia, other cranial neuropathies and lymphocytopenia are red flags enhancing early diagnostic suspicion. In Miller-Fisher Syndrome, ganglioside antibodies against GDlb, instead of QG1b, were found; because the COVID-19 spike protein also binds to sialic acid-containing glycoproteins for cell-entry and anti-GD $1 \mathrm{~b}$ antibodies typically cause ataxic neuropathy, cross-reactivity between COVID-19-bearing gangliosides and peripheral nerve glycolipids was addressed. Elevated Creatine Kinase $(>10,000)$ is reported in $10 \%$ of COVID-19-infected patients; two such patients presented with painful muscle weakness responding to IVIg indicating that COVID-19-triggered NAM is an overlooked entity. Cases of acute necrotizing brainstem encephalitis, cranial neuropathies with leptomeningeal enhancement, and tumefactive postgadolinium-enhanced demyelinating lesions are now emerging with the need to explore neuroinvasion and autoimmunity. Concerns for modifications-if any-of chronic immunotherapies with steroids, mycophenolate, azathioprine, IVIg, and anti-B-cell agents were addressed; the role of complement in innate immunity to viral responses and anti-complement therapeutics (i.e. eculizumab) were reviewed.

\section{Conclusions}

Emerging data indicate that COVID-19 can trigger not only GBS but other autoimmune neurological diseases necessitating vigilance for early diagnosis and therapy initiation. Although COVID-19 infection, like most other viruses, can potentially worsen patients with pre-existing autoimmunity, there is no evidence that patients with autoimmune neurological diseases stable on common immunotherapies are facing increased risks of infection.

\section{COVID-19 Resources}

For the latest articles, invited commentaries, and blogs from physicians around the world

NPub.org/COVID19 


\section{Glossary}

ACE-2 = angiotensin-converting enzyme 2; AIDP = acute inflammatory demyelinating polyneuropathy; AMAN = acute motor axonal neuropathy; $\mathbf{C K}=$ creatine kinase; $\mathbf{C R P}=\mathrm{C}$-reactive protein; $\mathbf{G B S}=$ Guillain-Barré syndrome; $\mathbf{I C U}=$ intensive care unit; IVIg = intravenous immunoglobulin; MERS = Middle East respiratory syndrome; MFS = Miller Fisher syndrome; NAM = necrotizing autoimmune myositis.

Guillain-Barré syndromes (GBSs) comprise a spectrum of polyneuropathies characterized by acute (within 1-4 weeks) ascending motor weakness, mild or moderate sensory abnormalities, occasional cranial nerve involvement, and muscle or radicular pain. According to the degree of involvement of the motor or sensory nerves, myelin sheath, axon, or cranial nerve predominance, the most common subtypes are the acute inflammatory demyelinating polyneuropathy (AIDP), acute motor axonal neuropathy (AMAN), and the Miller Fisher syndrome (MFS) characterized by acute ophthalmoplegia, gait ataxia, and areflexia. ${ }^{1}$ GBS is one of the prototypic viral-triggered autoimmune neurologic diseases as approximately $70 \%$ of the patients have a preceding by $1-3$ weeks, flu-like, viral illness. ${ }^{1-3}$ Among the infectious agents associated with triggering sporadic GBS are viruses, including influenza, enteroviruses, cytomegalovirus, Epstein-Barr virus, herpes simplex virus, hepatitis, or HIV, and bacteria, such as Campylobacter jejuni, Mycoplasma pneumoniae, and Haemophilus influenzae. ${ }^{1}$ GBS outbreaks have been also associated with viral epidemics or pandemics, including $\mathrm{H} 1 \mathrm{~N} 1$, swine flu A/New Jersey influenza strain, arthropod-borne flaviviruses, such as the West Nile virus, chikungunya, or Zika, and with coronaviruses, including the Middle East respiratory syndrome (MERS)-CoV and SARS-CoV. ${ }^{1-3}$

We are now more than 4 months into the peak of the COVID19 pandemic with $>5,000,000$ infections and $>330,000$ deaths steadily increasingly worldwide, and although various respiratory and cardiac complications have been reported, we have not yet seen COVID-19-related neuroinflammatory or neuroautoimmune diseases as with the other viral outbreaks, including with coronaviruses MERS-CoV and SARS-CoV, which have $75-80 \%$ identical viral genome sequence with COVID-19. ${ }^{4}$ Whether this is due to COVID-19-inducing severe respiratory compromise soon after the median 4-day incubation period ${ }^{5}$ and underrecognition of neurologic events owing to overwhelming urgency to focus on life-saving efforts is unclear. In the most up-to-date large published series, apart from multifactorial acute cerebrovascular events in $5.7 \%$ of patients, the main COVID-19-related neurologic symptoms have been hypogeusia (in 5.6\%), hyposmia (5.1\%), and very high creatine kinase (CK) levels, with myalgia (in 19.3\%) indicating potential CNS, peripheral nervous system, and myopathic manifestations. ${ }^{6}$ Things are however rapidly changing as just only the last 4 weeks the first COVID-19triggered neurologic events are reported with at least 11 cases of GBS, the prototypic viral-triggered autoimmune neurologic disease, observed in the 4 main COVID-19 hotspots, Wuhan,
Italy, Spain, and now France. ${ }^{7-11}$ The cases are of special neuroimmunologic and practical interest while highlight what is more to come.

The very first case - and the only one from Wuhan — refers to a woman who 4 days after returning to Shanghai from Wuhan presented with acute lower extremity weakness and areflexia that progressed over 3 days to the arms without any systemic symptoms. ${ }^{7}$ When first seen, GBS was suspected and confirmed by elevated CSF protein and electromyographic features of demyelinating neuropathy. Her laboratory test results were normal, except of lymphocytopenia and thrombocytopenia. She was started on intravenous immunoglobulin (IVIg) but 4 days later developed fever, cough, and pneumonia and tested COVID-19 positive. After 3 weeks, her strength normalized and lymphocytopenia resolved. While at the regular neurology ward, however, 2 of her relatives taking care of her tested COVID-19 positive with pneumonia, while 2 of her neurologists and 6 nurses treating her GBS were put on isolation.

Italy has just reported 6 patients with $\mathrm{GBS},{ }^{8} 1$ in this issue. ${ }^{9}$ All presented with subacute onset of upper and lower extremity weakness, distal paresthesias, and sensory deficits 3-10 days after experiencing cough, anosmia, ageusia, and sore throat. On admission, all had lymphocytopenia and high C-reactive protein (CRP) that led to diagnosis of COVID-19. One patient had facial diplegia and sensory ataxia. ${ }^{8}$ The CSF showed elevated protein concentration but was negative for COVID19. Electrophysiology was consistent with demyelinating or axonal GBS. MRI showed enhancement of the caudal nerve roots or facial nerve. Symptoms rapidly progressed to tetraplegia requiring mechanical ventilation. Antiganglioside antibodies in 3/6 tested patients were negative. All received IVIg with variable recovery; 1 died.

The third series refers to 2 men from Spain who, 3-5 days after experiencing low-grade fever, malaise, anosmia, and ageusia, developed MFS or polyneuritis cranialis. ${ }^{10}$ The patient with MFS presented with oculomotor nerve palsies, diplopia, perioral paresthesias, areflexia, ataxia, and elevated CSF protein concentration and the other with diplopia, bilateral abducens nerve palsy, and anosmia. Both had lymphopenia and tested positive for COVID-19; their SCF was COVID-19 negative. The patient with MFS was positive for GD1b ganglioside antibodies and improved with IVIg. In both, the neurologic features completely resolved, except for residual anosmia and ageusia. Last, 2 cases from France, a 43- 
year-old man and a 70-year-old woman, also presented with acute anosmia, followed by rapidly progressive weakness, paresthesias, ataxia, areflexia, and multiple cranial palsies (including III, V, VI, VII, and VIII), MRI enhancement of cranial nerves, roots, and plexus, high CSF protein concentration but negative for COVID-19, typical features of acute demyelinating neuropathy by electrophysiology, and clinical improvement after IVIg. ${ }^{11}$

\section{GBS in the COVID-19 pandemic}

These early GBS cases, despite incomplete immunologic workup considering the enormous difficulties in highly stressed in-hospital settings, provide early clues on what to expect in the months ahead regarding the common acute autoimmune neurologic conditions, such as GBS, polyneuritis cranialis, encephalitis, encephalomyelitis, or myositis, which for years we have been casually referring to as postviral if seen after febrile illnesses.

First and foremost, the practicing neurologists in this pandemic should now be aware that a patient who presents with an acute paralytic disease-like GBS, encephalomyelitis, or myositis-even without fever, dyspnea, or any systemic symptoms, may represent the first manifestation of COVID19. This is compelling, considering that only $43 \%$ of COVID19-positive patients on admission have fever, ${ }^{4-6}$ and many of the present patients with GBS did not have any COVID-19 symptoms at presentation. These early GBS cases also highlight that 2 clinical and laboratory signs, anosmia/ageusia and lymphocytopenia/thrombocytopenia, are red flags in suspecting COVID-19 in otherwise asymptomatic patients with acute neurologic events. They further confirm what we had feared from other viral pandemics that COVID-19 can trigger neurologic autoimmunity; in contrast to the other postviral neurologic diseases, however, COVID-19 requires high degree of suspicion as a potential hidden trigger to prevent inadvertent viral transmission to health care personnel and patient relatives, as in the Wuhan case. ${ }^{7}$ The series also highlights that GBS peaks 5-10 days after the first COVID-19 symptoms, which in intensive care unit (ICU) settings helps to distinguish GBS from critical illness neuropathy that usually appears later in the course of very sick ICU patients.

\section{Significance of anosmia/ageusia and other cranial nerve palsies}

The early manifestation of anosmia and ageusia not only in the present GBS series that often occurred in conjunction with other cranial neuropathies but also in large worldwide cohorts reporting sudden loss of smell and taste early in the infection in up to $60 \%$ of COVID-19 carriers ${ }^{5,6,12}$ is highly informative about COVID-19 neurovirulence or even possible viral entry into the brain. In contrast to commonly reversible anosmia when the non-neural olfactory epithelial cells are virally infected, the often persistent anosmia/ageusia after COVID-19 suggests neurotropism targeting olfactory neurons. SARS-CoV and MERS-CoV, the 2 coronaviruses similar to COVID-19, are neurovirulent and can enter the brain via olfactory nerves. ${ }^{13}$ In mice, after oronasal infection with SARS-CoV, the virus not only infects epithelial cells of the respiratory tract but also the olfactory receptor neurons in the neuroepithelium gaining access to the olfactory bulb and brainstem. ${ }^{13}$ These viruses can also enter the CNS via retrograde axonal transport through other cranial nerves, such as trigeminal, which possesses nociceptive neuronal receptors in the nasal cavity, the sensory fibers of the glossopharyngeal, and via peripheral nerves. ${ }^{13}$ The present GBS series, where oculomotor, trigeminal, and MRIenhanced facial and nerves roots were concurrently affected, strengthens (but not prove) this notion. Accordingly, it will not be unexpected in the weeks and months ahead to see other COVID-19-infected patients with neurologic signs related to multiple cranial nerves, brainstem, and peripheral nerves with MRI enhancement in nerves and meninges.

\section{Autoimmunity of COVID-19-GBS: significance of sialic acids present in the coronaviruses and peripheral} nerve myelin

In 7/11 tested patients with COVID-19-GBS, the virus was not detected in the CSF, implying no direct root infection or intrathecal viral replication. The improvement of several patients with IVIg and the presence of GD1b antibodies in 1 tested patient suggest a postviral-triggered immune response similar to other postviral-induced GBS cases ${ }^{1-3}$ or other postviral autoimmune neurologic disorders. ${ }^{14}$ The reported GD1b ganglioside antibodies, however, although in contrast to GQ1b antibodies typically seen in MFS, are of very special interest.

The SARS-CoV 3a protein contains oligosaccharides with direct evidence that sialic acids play a critical role in human coronavirus infection. ${ }^{15}$ It has been just shown that the attachment of coronaviruses to the surface of respiratory cells is mediated by the spike (S) viral protein, which binds not only to the angiotensin-converting enzyme 2 (ACE-2) receptor for entry ${ }^{16}$ but also to sialic acid-containing glycoproteins and gangliosides on cell surfaces. ${ }^{15}$ Such a dual receptor/ attachment is proposed to be a reason for the increased transmissibility of COVID-19 compared with SARS-CoV that binds only to ACE-2 receptor. ${ }^{15,16}$ Of relevance to GBS is that various gangliosides, most commonly those containing either a disialosyl moiety, such as GD1b, GQ1b, and GT1b, or 2 gangliosides that share epitopes with GM2, or a combination of GM2 and GM1, GM1 and GD1b, can serve as antigens in patients with neuropathies. ${ }^{17}$ When IgM recognizes the Gal (pl-3) GalNAc moiety of GM1, which is found on the surface of motor neurons, there is clinically a motor neuropathy, but if recognizes epitopes containing disialosyl groups of GDlb, which is present on the dorsal root ganglionic neurons, there is sensory ataxic neuropathy. ${ }^{17}$ Immunization of rabbits with 
GDlb also causes sensory ataxic neuropathy mimicking the human disease. ${ }^{18}$ Of interest, the first described patient with sensory ataxic neuropathy and GDlb antibodies had also ophthalmoplegia, ${ }^{19}$ as seen in MFS and the present series. ${ }^{10}$ As COVID-19 spike interacts with the GalNAc residue of GM1 and ganglioside dimers for anchoring to cell surface gangliosides, ${ }^{15}$ cross-reactivity between epitopes within the COVID-19 spike-bearing gangliosides and signature sugar residues of surface peripheral nerve glycolipids is a very likely possibility. Such typical molecular mimicry has been shown between peripheral nerve glycolipids and Campylobacter jejuni or Zika virus that also trigger GBS. ${ }^{1-3}$ Accordingly, all GBS subtypes (AIDP, AMAN, and MFS) can be expected with COVID-19, necessitating screening for ganglioside antibodies to assess autoimmunity. An interesting therapeutic component in this association is the emerging data that chloroquine, an antimalarial drug under investigation for treating COVID19, binds with high-affinity sialic acids and GM1 gangliosides and, in the presence of chloroquine, the SARS-CoV viral spike cannot bind gangliosides to infect the targeted cells. ${ }^{15}$ If benefit is confirmed and safety is established, chloroquine may be of added therapeutic value in future patients with COVID-19-triggered GBS in conjunction with IVIg.

\section{What is more to come with myositis in the offing}

Among the other potential COVID-19-associated autoimmune diseases, the first alarming concern is inflammatory myopathy, especially necrotizing autoimmune myositis (NAM) because very high CK levels $>10,000$ with myalgia and weakness are now reported in more than 10\% of COVID19-infected patients. ${ }^{6}$ Although COVID-19-associated myopathy has not yet been studied but only characterized as skeletal muscle injury or rhabdomyolysis, ${ }^{6} 2$ just published cases suggest an autoimmune COVID-19-triggered NAM. One, an 88-year-old man from New York presented with acute bilateral thigh weakness and inability to get up from the toilet, without fever or other systemic symptoms, and very high CK level $(13,581 \mathrm{U} / \mathrm{L}) .{ }^{20} \mathrm{He}$ was found COVID-19 positive and given hydroxychloroquine, and a week later, his painful weakness improved with CK reduction. The other, a 60-year-old man from Wuhan had a 6-day history of fever, cough, and COVID-19-positive pneumonia with normal strength and CK; 7 days later, although systemically had improved, his CRP doubled and developed painful muscle weakness with very high $\mathrm{CK}(11,842 \mathrm{U} / \mathrm{L}) .{ }^{21} \mathrm{He}$ was given IVIg and his strength improved while became COVID-19 negative.

Myopathic symptoms in a severe systemic viral disease are multifactorial, but an acute onset of severe muscle weakness with increased inflammatory markers and very high CK levels in the thousands, as described above, is consistent with autoimmune inflammatory myopathy within the spectrum of NAM, similar to what was first reported with HIV early in that epidemic. $^{22,23}$ The most common causes of NAM are not chronic statin use as overstated, but autoimmune, paraneoplastic, or postviral. ${ }^{22-25}$ Considering that very high CK level and painful muscle weakness were seen in 10\% of COVID19-positive patients, ${ }^{6}$ a potentially treatable autoimmune myopathy has been likely overlooked. Studies with muscle biopsy and antibody screening are urgently needed in such patients because therapy with IVIg can improve strength, reduce morbidity, and facilitate recovery, as reported in the first 2 cases. ${ }^{20,21}$ COVID-19-associated myositis is also highly interesting because ACE-2, the SARS-CoV receptor, is reportedly expressed in skeletal muscle. ${ }^{26}$ If this is confirmed, COVID-19 may represent the first virus directly capable of infecting muscle fibers. None of the viruses implicated in viral-triggered myositis has so far been shown to infect muscle ${ }^{27,28}$; instead, viruses induce an immune $T$ cell with clonal expansion of viral-specific $\mathrm{T}$ cells or macrophage-mediated, muscle fiber autoinvasion with abundant proinflammatory cytokines. ${ }^{22,29,30}$

\section{Other emerging postinfectious autoimmune neurologic disorders}

Acute disseminated encephalomyelitis, as described for the other coronaviruses, or postinfectious brainstem encephalitis, myelitis, and radiculoneuropathies will not be unexpected. An atypical case of acute necrotizing encephalopathy, attributed to immune-mediated process and proinflammatory cytokines, and another with meningoencephalitis and leptomeningeal enhancement have been already noted. ${ }^{31}$ This picture is now becoming more clear with 2 reports in this issue of the Journal, documented by impressive MRIs; one, a case of acute necrotizing brainstem encephalopathy, presented with epilepsy and changes in the right mesial temporal lobe and hippocampus ${ }^{32}$ and another with altered mental status and multiple white matter tumefactive lesions with postgadolinium enhancement in periventricular areas and corpus callosum suggestive of demyelination..$^{33}$ Not underestimating the overwhelming burden of acute COVID-19 to medical staff, vigilance for these disorders is needed along with screening for autoimmune encephalitis autoantibodies ${ }^{14}$ because these cases can potentially respond to early initiation of immunotherapy, especially with IVIg, whenever indicated, which may additionally offer various potentially protective antibodies and anticytokine effects.

\section{Residual neurologic deficits in COVID-19-recovered patients: need for a systematic study}

No neurologic data are yet available about the discharged patients who survived the catastrophic effects of the virus. We know that several people have permanently lost smell and taste, which is a form of disability affecting quality of life, depriving tasting pleasures, and the ability to detect danger signals, like smelling gas or fire. Many discharged patients require assistance because of muscle weakness and gait unsteadiness, which is arguably multifactorial; some patients may have had critical 
illness neuropathy and deconditioning with significant muscle atrophy worsened by comorbidities; others may have neurotoxicity or myocytotoxicity from antiviral therapies, like first described with antiretrovirals or chloroquine ${ }^{34-36}$; still others may have the residual effects from an unrecognized primary myopathy, neuropathy, or myelopathy due to postviral autoimmunity. A study exploring the patients' current causes of residual muscle weakness and sensory deficits is urgently needed using EMG, muscle or nerve biopsies, autoantibody screening, and CSF or imaging studies to determine immediate or long-term recovery prospects, identify potential reversibility, and accelerate return to normalcy.

\section{Patients with autoimmune neurologic diseases: how is COVID-19 changing ongoing immunotherapies and the role of complement}

Patients with autoimmune neuropathies, especially chronic inflammatory demyelinating polyneuropathy and multifocal motor neuropathy, but also with other autoimmunities like myasthenia gravis, MS, and inflammatory myopathies, have been justifiably concerned as to whether their disease status adds an additional risk placing them into an immunosuppressed or immunocompromised category. There is no current evidence that any of the aforementioned autoimmune disorder itself makes them more susceptible to COVID-19, but some immunosuppressive or even immunomodulating therapies they are receiving may have this potential, although there are no validated data. Most autoimmune neuromuscular patients are maintained on steroids, mycophenolate, or azathioprine while most chronic inflammatory demyelinating polyneuropathy receive monthly IVIg. The same applies to patients with MS where it seems safe to start or continue treatment with the standard disease-modifying drugs. If clinically stable and not lymphopenic, there are no compelling or data-driven reasons to change anything in these patients and disturb clinical stability. For patients on monthly IVIg, there may be even a theoretical advantage that IVIg offers additional protection due to natural autoantibodies; if IVIg is not infused as home infusion, switching to self-administered subcutaneous IgG might be an option to diminish exposure, as has been proven effective. ${ }^{37}$ For patients on rituximab, the infusion intervals can be prolonged to more than 6 months because both B-cell reduction and clinical benefit can persist longer. ${ }^{38} \mathrm{New}$ emerging data provide credence and reassurance regarding these issues, especially on immunomodulating drugs. In a large number of patients from Wuhan, published in this issue of the Journal, ${ }^{39}$ it was shown that altered immunity induced by diseasemodifying drugs in patients with MS or neuromyelitis optica spectrum disorder appears insufficient to enhance susceptibility to COVID-19 infection. The results are important but not unexpected considering that most of these therapies target the adaptive immune response with little, if any, effect on suppressing the innate immunity that facilitates infection of macrophages and viral spread. Similar data from New York City area show that the incidence of hospitalization among patients with immune-mediated inflammatory diseases on therapy with steroids and biologic agents was consistent with that among patients with COVID-19 in the general population, concluding that ongoing use of biologics is not associated with worse COVID-19 outcomes. ${ }^{40}$ There is also new evidence suggesting possible beneficial effect of anticomplement therapies. ${ }^{41}$ Complement is an integral component of the innate immune response to viruses and an instigator of proinflammatory responses with evidence that activation of $\mathrm{C} 3$ exacerbates SARS-CoV-associated acute respiratory distress syndrome and C3-C5 complement deposits are abundant in the lung biopsies from patients with COVID$19 .{ }^{41}$ It was proposed that complement inhibition may alleviate the inflammatory complications of COVID-19 infection leading to ongoing trials with anti-C3 and anti-C5 agents. ${ }^{41,42}$ On this basis, eculizumab, an anti-C5 monoclonal antibody approved for neuromyelitis optica spectrum disorder and myasthenia gravis with some benefits in patients with GBS, may not be withheld, if indicated, as, like IVIg, may theoretically have added benefit.

\section{Study funding}

No targeted funding reported.

\section{Disclosure}

M. Dalakas is an associate editor for Neurology: Neuroimmunology \& Neuroinflammation. Disclosures available: Neurology.org/NN.

\section{Publication history}

Received by Neurology: Neuroimmunology \& Neuroinflammation May 1, 2020. Accepted in final form May 4, 2020.

\section{References}

1. Dalakas MC. Pathogenesis of immune-mediated neuropathies Biochimica et. Biophyiscal Acta 2015;1852:658-666.

2. Wakerley BR, Yuki N. Infectious and noninfectious triggers in Guillain-Barre syndrome. Expert Rev Clin Immunol 2013;9:627-639.

3. Cao-Lormeau VM, Blake A, Mons S, et al. Guillain-Barre syndrome outbreak associated with Zika virus infection in French Polynesia: a case-control study. Lancet 2016;387:1531-1539.

4. Zhu N, Zhang D, Wang W, et al. A novel coronavirus from patients with pneumonia in China, 2019. N Engl J Med 2020;382:727-733.

5. Guan WJ, Ni ZY, Hu Y, et al. Clinical characteristics of coronavirus disease 2019 in China. N Engl J Med 2020;382:1708-1720.

6. Mao L, Jin H,Wang M, et al. Neurologic manifestations of hospitalized patients with coronavirus disease 2019 in Wuhan, China. JAMA Neurol 2020. 10.1001/jamaneurol. 2020.1127 .

7. Zhao H, Shen D, Zhou H, Liu J, Chen S. Guillain-Barré syndrome associated with SARS-CoV-2 infection: causality or coincidence? Lancet Neurol 2020;19: 383-384.

8. Toscano G, Palmerini F, Ravaglia S, et al. Guillain-Barré syndrome associated with SARS-CoV-2. New Engl J Med 2020. 10.1056/NEJMc2009191.

9. Alberti P, Beretta S, Piatti M et al. Guillain-Barré syndrome related to COVID-19 infection. Neurol Neuroimmunol Neuroinflamm 2020;7:e741. doi: 10.1212/ NXI.0000000000000741.

10. Gutiérrez-Ortiz C, Méndez A, Rodrigo-Rey S, et al. Miller Fisher Syndrome and polyneuritis cranialis in COVID-19. Neurology Epub 2020 Apr 17. doi: 10.1212/ WNL.0000000000009619.

11. Bigaut K, Mallaret M, Baloglu S, et al. Guillain-Barré syndrome related to SARS-CoV2 infection. Neurol Neuroimmunol Neuroinflamm 2020;7:e785. doi: 10.1212/ NXI.0000000000000785. 
12. Yan CH, Faraji F, Ptajapati D, DeConde AS. Association of chemosensory dysfunction and covid-19 in patients presenting with influenza-like symptoms. Int Forum Allergy Rhinology Epub 2020 Apr 12. doi: 10.1002/alr.22579.

13. Desforges $\mathrm{M}$, Coupanec A, Dubeau P, et al. Human coronaviruses and other respiratory viruses: underestimated opportunistic pathogens of the central nervous system. Viruses 2019;12:14.

14. Armangue T, Spatola M, Vlagea A, et al. Frequency, symptoms, risk factors, and outcomes of autoimmune encephalitis after herpes simplex encephalitis: a prospective observational study and retrospective analysis. Lancet Neurol 2018;17:760-772.

15. Fantini J, Di Scala C, Chahinian H, Yahi N. Structural and molecular modeling studies reveal a new mechanism of action of chloroquine and hydroxychloroquine against SARSCoV-2 infection. Int J Antimicrob Agents 2020. 10.1016/j.ijantimicag.2020.105960.

16. Vaduganathan M, Vardeny $\mathrm{O}$, Michel T, et al. Renin-angiotensin-aldosterone system inhibitors in patients with covid-19. N Engl J Med 2020;382:1653-1659.

17. Dalakas MC. Quarles RH. Autoimmune ataxic Neuropathies (sensory ganglionopathies): are glycolipids the responsible autoantigens? Ann Neurol 1996;39:419-422.

18. Kusunoki S, Shimizu J, Chiba A, et al. Experimental sensory neuropathy induced by sensitization with ganglioside GDlb. Ann Neurol 1996;39:424-431.

19. Daune GC, Farrer RG, Dalakas MC, Quarles RH. Sensory neuropathy associated with monoclonal IgM to GD1b ganglioside. Ann Neurol 1992;31:683-685.

20. Suwanwongse K, Shabarek N. Rhabdomyolysis as a presentation of 2019 novel coronavirus disease. Cureus 2020;12:e7561.

21. Jin M, Tong Q. Rhabdomyolysis as potential late complication associated with 2019 novel coronavirus disease. Emerg Infect Dis 2020;26.

22. Dalakas MC. Inflammatory muscle diseases. N Engl J Med 2015;372:1734-1747.

23. Dalakas MC, Pezeshkpour GH, Gravell M, Sever JL. Polymyositis associated with AIDS retrovirus. JAMA 1986;256:2381-2383.

24. Dalakas MC. Are autoantibodies pathogenic in necrotizing myopathy? Nat Rev Rheumatol 2018;14:251-252.

25. Watanabe Y, Uruha A, Suzuki S, et al. Clinical features and prognosis in anti-SRP and antiHMGCR necrotizing myopathy. J Neurol Neurosurg Psychiatry 2016;87:1038-1044.

26. Cabello-Verrugio C, Morales M, Rivera J, et al. Renin-angiotensin system: an old player with novel functions in skeletal muscle. Med Res Rev 2015;35:437-463.

27. Leff RL, Love LA, Miller FW, et al. Viruses in the idiopathic inflammatory myopathies: absence of candidate viral genomes in muscle. Lancet 1992;339:1192-1195.

28. Leon-Monzon M, Lamperth L, Dalakas MC. Search for HIV proviral DNA and amplified sequences in the muscle biopsies of patients with HIV polymyositis. Muscle Nerve 1993;16:408-413.
29. Illa I, Nath A, Dalakas MC. Immunocytochemical and virological characteristics of HIV-associated inflammatory myopathies: similarities with seronegative polymyositis. Ann Neurol 1991;29:474-481.

30. Dalakas MC, Rakocevic G, Shatunov A, et al. Inclusion body myositis with human immunodeficiency virus infection: four cases with clonal expansion of viral-specific T cells. Ann Neurol 2007;61:466-475.

31. Moriguchi T, Harii N, Goto J, et al. A first Case of Meningitis/Encephalitis associated with SARS-Coronavirus-2. Int J Infect Dis 2020;94:55-58.

32. Dixon L, Varley J, Gontsarova A, et al. COVID-19-related acute necrotizing encephalopathy with brain stem involvement in a patient with aplastic anemia. Neurol Neuroimmunol Neuroinflamm 2020;7:e789. doi: 10.1212/NXI.0000000000000789.

33. Brun G, Hak JF, Coze S, et al. COVID-19-White matter and globus pallidum lesions: Demyelination or small-vessel vasculitis? Neurol Neuroimmunol Neuroinflamm 2020;7:e777. doi: 10.1212/NXI.0000000000000777.

34. Dalakas MC, Illa I, Pezeshkpour GH, Laukaitis JP, Cohen B, Griffin J. Mitochondrial myopathy caused by long-term zidovudine therapy. N Engl J Med 1990;332: 1098-1105.

35. Dalakas MC. Peripheral neuropathy and antiretroviral drugs. J Peripher Nerv Syst 2001;6:14-20.

36. Stein M, Bell MJ, Ang LC. Hydroxychloroquine neuromyotoxicity. J Rheumatol 2000; 27:2927-2931.

37. van Schaik IN, Bril V, van Geloven N, et al; For the PATH Study Group. Subcutaneous immunoglobulin for maintenance treatment in chronic inflammatory demyelinating polyneuropathy (PATH): a randomised, double-blind, placebocontrolled, phase 3 trial. Lancet Neurol 2017;17:35-46.

38. Dalakas MC. Inhibition of B cell functions: implications for neurology. Neurology 2008;70:2252-2260.

39. Fan M, Qiu W, Bu B, et al. Risk of COVID-19 infection in MS and neuromyelitis optica spectrum disorders. Neurol Neuroimmunol Neuroinflamm 2020;7:e787. doi: 10.1212/NXI.0000000000000787.

40. Habernan R, Axelrad J, Chen A, et al. Covid-19 in immune-mediated inflammatory diseases—case series from New York. 10.1056/NEJMc2009567.

41. Risitano AM, Mastellos DC, Huber-Lang M, et al. Complement as a target in COVID19 ? Nat Rev Immunol 2020. 10.1038/s41577-020-0320-7.

42. Eculizumab (Soliris) in Covid-19 Infected Patients (SOLID-C19). Available at: https://clinicaltrials.gov/ct2/show/NCT04288713. NLM identifier: NCT04288713. Accessed April 27, 2020 


\title{
Neurology \\ Neuroimmunology \& Neuroinflammation
}

\author{
Guillain-Barré syndrome: The first documented COVID-19-triggered autoimmune \\ neurologic disease: More to come with myositis in the offing \\ Marinos C. Dalakas \\ Neurol Neuroimmunol Neuroinflamm 2020;7; \\ DOI 10.1212/NXI.0000000000000781
}

This information is current as of June 9, 2020

Neurol Neuroimmunol Neuroinflamm is an official journal of the American Academy of Neurology.

Published since April 2014, it is an open-access, online-only, continuous publication journal. Copyright

Copyright $\left({ }^{\circ} 2020\right.$ The Author(s). Published by Wolters Kluwer Health, Inc. on behalf of the American

Academy of Neurology.. All rights reserved. Online ISSN: 2332-7812.

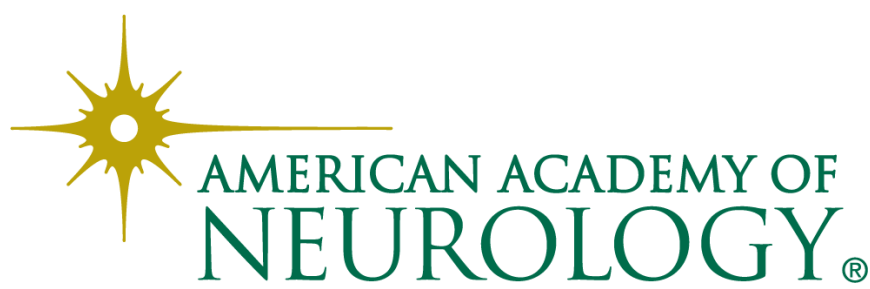




\section{Updated Information \& Services}

References

Citations

Subspecialty Collections

Permissions \& Licensing

Reprints including high resolution figures, can be found at: http://nn.neurology.org/content/7/5/e781.full.html

This article cites 35 articles, 8 of which you can access for free at: http://nn.neurology.org/content/7/5/e781.full.html\#\#ref-list-1

This article has been cited by 20 HighWire-hosted articles: http://nn.neurology.org/content/7/5/e781.full.html\#\#otherarticles

This article, along with others on similar topics, appears in the following collection(s):

All Immunology

http://nn.neurology.org//cgi/collection/all_immunology COVID-19

http://nn.neurology.org//cgi/collection/covid_19

Cranial neuropathy

http://nn.neurology.org//cgi/collection/cranial_neuropathy

Guillain-Barre syndrome

http://nn.neurology.org//cgi/collection/guillainbarre_syndrome

Muscle disease

http://nn.neurology.org//cgi/collection/muscle_disease

Information about reproducing this article in parts (figures,tables) or in its entirety can be found online at:

http://nn.neurology.org/misc/about.xhtml\#permissions

Information about ordering reprints can be found online:

http://nn.neurology.org/misc/addir.xhtml\#reprintsus

Neurol Neuroimmunol Neuroinflamm is an official journal of the American Academy of Neurology.

Published since April 2014, it is an open-access, online-only, continuous publication journal. Copyright

Copyright $\odot 2020$ The Author(s). Published by Wolters Kluwer Health, Inc. on behalf of the American

Academy of Neurology.. All rights reserved. Online ISSN: 2332-7812.

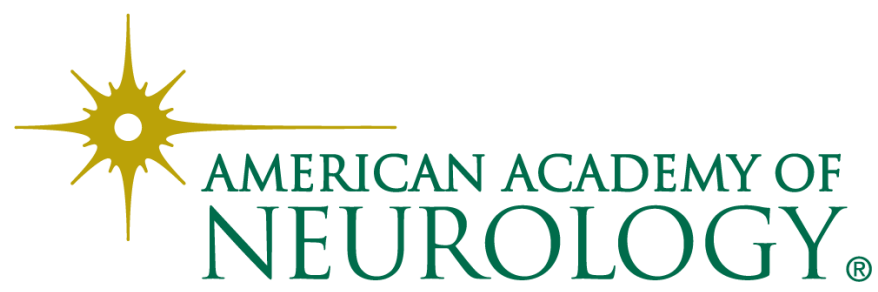

